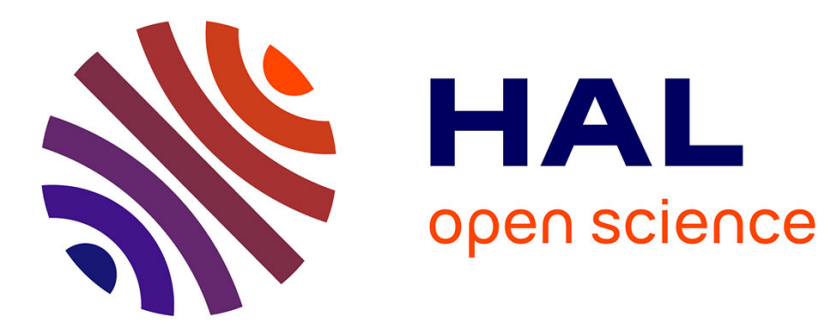

\title{
Control design for discrete-time bilinear systems using the scalarized Schur complement
}

\author{
Mohsen Vatani, Morten Hovd, Sorin Olaru
}

\section{To cite this version:}

Mohsen Vatani, Morten Hovd, Sorin Olaru. Control design for discrete-time bilinear systems using the scalarized Schur complement. International Journal of Robust and Nonlinear Control, 2017, 27 (18), pp.4492 - 4506. 10.1002/rnc.3807 . hal-01719796

\section{HAL Id: hal-01719796 \\ https://hal-centralesupelec.archives-ouvertes.fr/hal-01719796}

Submitted on 6 Mar 2020

HAL is a multi-disciplinary open access archive for the deposit and dissemination of scientific research documents, whether they are published or not. The documents may come from teaching and research institutions in France or abroad, or from public or private research centers.
L'archive ouverte pluridisciplinaire HAL, est destinée au dépôt et à la diffusion de documents scientifiques de niveau recherche, publiés ou non, émanant des établissements d'enseignement et de recherche français ou étrangers, des laboratoires publics ou privés. 
archives-ouvertes

\title{
Control design for discrete-time bilinear systems using the scalarized Schur complement
}

\author{
Mohsen Vatani, Morten Hovd, Sorin Olaru
}

\section{To cite this version:}

Mohsen Vatani, Morten Hovd, Sorin Olaru. Control design for discrete-time bilinear systems using the scalarized Schur complement. International Journal of Robust and Nonlinear Control, Wiley, 2017, 27 (18), pp.4492 - 4506. 10.1002/rnc.3807 . hal-01719796

\section{HAL Id: hal-01719796 \\ https://hal-centralesupelec.archives-ouvertes.fr/hal-01719796}

Submitted on 6 Mar 2020

HAL is a multi-disciplinary open access archive for the deposit and dissemination of scientific research documents, whether they are published or not. The documents may come from teaching and research institutions in France or abroad, or from public or private research centers.
L'archive ouverte pluridisciplinaire HAL, est destinée au dépôt et à la diffusion de documents scientifiques de niveau recherche, publiés ou non, émanant des établissements d'enseignement et de recherche français ou étrangers, des laboratoires publics ou privés. 


\title{
Control design for discrete time bilinear systems using the scalarized Schur complement
}

\author{
M. Vatani ${ }^{1}$, M. Hovd ${ }^{1 *}$ and S. Olaru ${ }^{2}$ \\ ${ }^{1}$ Engineering Cybernetics Department, Norwegian University of Science and Technology, N-7491 Trondheim, Norway \\ ${ }^{2}$ CentraleSupelec, Automatic Control Department and EPI INRIA DISCO, 3 rue Joliot Curie, 91192 France
}

\begin{abstract}
SUMMARY
In this paper, controller design for discrete time bilinear systems is investigated by using Sum of Squares (SOS) programming methods and quadratic Lyapunov functions. The class of rational polynomial controllers are considered, and necessary conditions on the degree of controller polynomials for quadratic stability are derived. Next, a scalarized version of the Schur complement is proposed. For controller design, the Lyapunov difference inequality is converted to a SOS problem, and an optimization problem is proposed to design a controller which maximizes the region of quadratic stability of the bilinear system. Input constraints can also be accounted for.
\end{abstract}

Copyright $\bigcirc 2010$ John Wiley \& Sons, Ltd.

Received ...

KEY WORDS: Discrete-time bilinear systems, sum of squares programming, scalarized Schur

\section{INTRODUCTION}

Since the work of Parrilo [1] there have been considerable advances on analysis and controller design using Sum of Squares (SOS) programming. In [2] a general framework using Sum of Squares (SOS) programming for analyzing nonlinear systems stability is developed for continuous-time systems. An extensive exposition of the use of SOS programming for controller design and domain of attraction analysis for continuous time systems is given in [3]. Use of SOS programming for the design of polynomial controllers for polynomial continuous-time systems is studied in [4] and [5], while works on nonlinear discrete-time systems include, e.g., [6], [7]. In [7] the use of linear state feedback is studied, whereas [6] addresses the synthesis of polynomial controllers, taking input saturation into account. This paper considers SOS based controller design for discrete-time bilinear systems using rational polynomial controllers.

*Correspondence to: Engineering Cybernetics Department, Norwegian University of Science and Technology, N-7491

Trondheim, Norway. E-mail: morten.hovd@itk.ntnu.no 
Bilinear systems are a subclass of polynomial input affine systems, where the nonlinearity consists of products between the states and inputs. Although the class of bilinear systems have properties which make them 'close' to the class of linear systems, linearization results in neglecting the main challenge in controller design for these systems. Bilinear systems find many practical applications in various fields (for example power systems [8] or the control in intelligent buildings [9]), and many nonlinear systems could be approximated by bilinear models.

A substantial number of works have been devoted to control and analysis of continuous-time bilinear systems. A representative overview of these works is beyond the scope of this paper, but some inspiration from Gutman [10] is acknowledged. Closer to the topic of interest of the current paper, controller design using SOS programming for continuous time bilinear systems has previously been addressed in $[11,12]$. There are fewer references on discrete time bilinear systems. In his 2009 book on bilinear control systems, Elliott [13] devotes one chapter to discrete-time systems, whereas the book by Pardalos and Yatsenko [14] considers continuous time exclusively. An important work specifically addressing discrete-time bilinear systems is that of Lin and Byrnes [15], who design a globally stabilizing controller for passive bilinear systems. In [16] a nonlinear state feedback control has been proposed to asymptotically stabilize a neutrally stable system. In [17] robustly stabilizing controllers for singularly perturbed, open loop stable discrete time bilinear systems with a single input are proposed. The nominal controller designs in [17] are extended to multivariable systems in [18], again for open loop stable systems. Lu et al. [19] considers global stabilization of neutrally stable discrete-time bilinear descriptor systems while accounting for input saturation. Tang and coworkers [20] study optimal control of bilinear discrete-time systems with a quadratic performance criterion, and develop a controller requiring the on-line solution of a twopoint boundary value problem.

Model Predictive Control of discrete time bilinear systems is studied in, e.g., [21], [22]. References [23] and [24] investigate the constrained and unconstrained stabilization of discrete time bilinear systems using polyhedral Lyapunov functions. The results are further developed in [25] to handle discrete bilinear system with additive bounded disturbances.

From a structural point of view, it can be noted that several authors, (e.g. [15], [26]) have proposed controllers for discrete-time bilinear systems that take the form of ratios of polynomials. In this paper it will be shown that under specified conditions on the bilinear system structure, global quadratic stability of open loop unstable discrete-time bilinear systems will require an open loop unstable state to have the same maximal degree in the numerator and denominator polynomials of the controller. Subsequently, a controller design procedure based on SOS programming will be developed.

To the best of the authors' knowledge, this is the first work specifically addressing the quadratic stabilization of discrete time bilinear systems using SOS programming, with the exception of our previous work [27] which presents some preliminary results. The present work significantly extends the results of [27]. Unlike the designs in [15-19,26], the design procedure developed here can handle both open loop unstable systems and systems with multiple inputs. The design results in rational polynomial controllers, with low online computational complexity compared to the control proposed by Tang [20] and MPC-based approaches [21,22]. The results in Section 5 indicate that a larger stable region is achieved than what is obtained in [24]. Although the resulting computational problems at the design stage are relatively complex, it is found that software for SOS programming 
are now of a quality that makes this technique useful and relatively accessible. The software package YALMIP $[28,29]$ has been used for all SOS problems in this paper.

This paper is organized as follows: In Section 2, the problem is defined and preliminary information is provided. Section 3 proposes to calculate the input as the ratio of two polynomials in the states, and observations regarding the degrees of these polynomials with regards to global quadratic stability are made. In Section 4 the proposed controller design method is presented. Section 5 provides illustrative examples. The paper ends with a brief conclusion section.

\section{Notation and definitions}

A norm of a of a real vector in $\mathbb{R}^{n}$ is denoted by the symbol $\|$.$\| . A function \psi: \mathbb{R}_{+} \rightarrow \mathbb{R}_{+}$is said to be of class $\mathcal{K}$ if it is continuous, zero at the origin and strictly increasing. A class $\mathcal{K}$ function is called $\mathcal{K}_{\infty}$ if it is also unbounded.

A function $\phi: \mathbb{R}^{n} \rightarrow \mathbb{R}$ is positive semidefinite if $\phi(x) \geq 0, \forall x \in \mathbb{R}^{n}$. If $\phi(x)>0 \forall x \neq 0$ then the function is called positive definite. The function $f(x)$ is negative definite if $-f(x)$ is positive definite.

Consider a discrete time system $x(k+1)=f(x(k))$ with a fixed point $f(0)=0$.

\section{Definition 1}

A set $S \subset \mathbb{R}^{n}$ is positive invariant with respect to the discrete-time dynamics $x(k+1)=f(x(k))$ if for all $x \in S$ it holds that $f(x) \in S$.

Given a positive invariant set $D \subseteq \mathbb{R}^{n}$ with the origin in its interior, a function $V():. D \rightarrow \mathbb{R}$ with $V(0)=0$ is a Lyapunov function if there exist $W_{1}, W_{2} \in \mathcal{K}_{\infty}$ such that:

$$
W_{1}(\|x\|) \leq V(x) \leq W_{2}(\|x\|), \quad \forall x \in D
$$

and the rate of change $V(f(x))-V(x)<0, \forall x \in D \backslash\{0\}$. The existence of a Lyapunov function guarantees the asymptotic stability of the origin for any initial state in $D$.

\section{Definition 2}

Given the discrete time system $x(k+1)=f(x(k))$ with a fixed point $f(0)=0$, the set of all initial conditions $x(0) \in \mathbb{R}^{n}$ for which the trajectories converge to the origin is called the domain of attraction.

This paper will focus on controller design for ensuring stability inside a sublevel set of the Lyapunov function. A sublevel set of a Lyapunov function is by definition positive invariant [30], and is a subset of the domain of attraction of the origin.

\section{Definition 3}

The system $x(k+1)=f(x(k))$ with a fixed point $f(0)=0$ is quadratic Lyapunov stable if there exists a matrix $P>0$ defining a Lyapunov function $V(x)=x^{T} P x$ and the domain $D=\{x \in$ $\left.\mathbb{R}^{n} \mid x^{T} P x \leq \gamma\right\}$ will define a positive invariant set for a positive constant $\gamma$. 


\section{PROBLEM STATEMENT AND PRELIMINARIES}

This paper considers the control of discrete-time bilinear systems:

$$
x(k+1)=A x(k)+\sum_{i=1}^{m}\left(B_{i} x(k)+b_{i}\right) u_{i}(k)
$$

where $x(k) \in \mathbb{R}^{n}$ is the state vector at time $k, u(k) \in \mathbb{R}^{m}$ is the input vector at time $k$ and $u_{i}(k)$ is the $i$-th element of input vector, while $A \in \mathbb{R}^{n \times n}, B_{i} \in \mathbb{R}^{n \times n}, b_{i} \in \mathbb{R}^{n \times 1}$ are matrices. It is assumed that the origin is an equilibrium point of the autonomous system. For the sake of simplicity of notation, (2) is reformulated as:

$$
x(k+1)=A x(k)+\left(B_{x}+B\right) u(k)
$$

where $B_{x}=\left[B_{1} x(k) B_{2} x(k) \cdots B_{m} x(k)\right]$ and $B=\left[b_{1} b_{2} \cdots b_{m}\right]$. In expressions where no confusion can arise, and all states have the same time index $k$, the time index may be dropped for simplicity.

Of particular interest here are necessary and sufficient conditions for quadratic stability when using rational polynomial controllers:

$$
u_{i}(x)=\frac{c_{i}(x)}{c_{0}(x)}
$$

where $c_{i}(x)$ are polynomials in the state with lowest degree one and highest degree $n_{n}$, and $c_{0}(x)$ is a polynomial of lowest degree zero and highest degree $n_{d}$. All inputs share the same denominator polynomial $c_{0}(x)$. Note that for a given $x$, these polynomials are linear in the polynomial coefficients $\left(c_{c i}\right)$, an important fact when optimizing over polynomial coefficients in the controller design. While the assumption of a common denominator polynomial might seem restrictive, this is in fact not so, as the common denominator polynomial can be chosen as the least common multiple of the denominator polynomials for the individual inputs.

For controller design, SOS methods are exploited in the present paper. The basic idea behind the SOS approach for checking the positivity of a polynomial $p(x)$, is to replace the positivity with the condition that the polynomial can be transformed to a sum of squares [1]:

$$
p(x)=\sum_{i=1}^{N} h_{i}^{2}(x)=\sum_{i=1}^{M}\left(q_{i}^{T} v(x)\right)^{2}=v^{T}(x) Q v(x)
$$

where $Q=Q^{T}>0$. As the result, if it is possible to find a vector of monomials $v(x)$ and a positive definite matrix $Q$, positivity of $p(x)$ is guaranteed. Similarly, a symmetric polynomial matrix $M(x)$ is said to be an SOS matrix if it can be decomposed into

$$
M(x)=H^{T}(x) H(x)
$$

The SOS decomposition can be computed by semi-definite programming with the help of available software [29]. 


\section{THE FUNCTIONAL FORM OF THE CONTROLLER AND REQUIREMENTS FOR GLOBAL ASYMPTOTIC STABILITY}

For bilinear systems with a diagonalizable matrix $A$, a change of coordinates can be performed in order to obtain an equivalent state vector $\tilde{x}$, transforming (3) to

$$
\tilde{x}(k+1)=\Lambda \tilde{x}(k)+\left(\tilde{B}_{\tilde{x}}+\tilde{B}\right) u(k)
$$

where $\Lambda=\operatorname{diag}\left(\lambda_{j}\right)$ is the eigenvalue matrix of $A$. Similarly, the controller polynomials $c_{i}(x)$ and $c_{0}(x)$ may equivalently be expressed as $\tilde{c}_{i}(\tilde{x})$ and $\tilde{c}_{0}(\tilde{x})$, respectively.

\section{Definition 4}

The dynamical mode represented by the state $\tilde{x}_{j}$ corresponding to eigenvalue $\lambda_{j}$ in (7) is called a linear mode if row $j$ of $\tilde{B}_{\tilde{x}}$ is zero. All modes that are not linear are bilinear modes. The mode represented by the state $\tilde{x}_{j}$ is called endogenously bilinear if row $j$ of $\tilde{B}_{\tilde{x}}$ exhibits linear dependence on $\tilde{x}_{j}$ (irrespective of possible linear dependencies on other states $\tilde{x}_{i}, i \neq j$ ).

\section{Proposition 1}

Consider a single-input bilinear discrete time system of the form (7) and a rational polynomial controller (4). The closed loop system is globally quadratic stable only if any state $\tilde{x}_{j}$ representing an endogenously bilinear mode has the same maximal degree in the numerator and denominator polynomial of the rational polynomial controller.

Proof: Without loss of generality, assume that $j=1$. The proposition will be decomposed into two statements:

1. The maximal degree of $\tilde{x}_{1}$ in the denominator must be at least as high as the maximal degree of $\tilde{x}_{1}$ in the numerator.

2. The maximal degree of $\tilde{x}_{1}$ in the numerator must be at least as high as the maximal degree of $\tilde{x}_{1}$ in the denominator.

For point 1), consider the Lyapunov difference inequality $V(f(\tilde{x}))-V(\tilde{x})<0$ for the quadratic Lyapunov function $V(\tilde{x})=\tilde{x}^{T} \tilde{P} \tilde{x}$. Substitute in the plant dynamics (7), the controller (4), and multiply with $\tilde{c}_{0}(\tilde{x})^{2}$ to obtain

$$
\left(\Lambda \tilde{x} \tilde{c}_{0}(\tilde{x})+\left(\tilde{B}_{\tilde{x}}+\tilde{B}\right) \tilde{c}_{1}(\tilde{x})\right)^{T} \tilde{P}\left(\Lambda \tilde{x} \tilde{c}_{0}(\tilde{x})+\left(\tilde{B}_{\tilde{x}}+\tilde{B}\right) \tilde{c}_{1}(\tilde{x})\right)-\tilde{c}_{0}(\tilde{x}) \tilde{x}^{T} \tilde{P} \tilde{x} \tilde{c}_{0}(\tilde{x})<0
$$

If the maximal degree of $\tilde{x}_{1}$ in $\tilde{c}_{1}(\tilde{x})$ is higher than the maximal degree of $\tilde{x}_{1}$ in $\tilde{c}_{0}(\tilde{x})$, the first term of the Lyapunov difference inequality will be of higher degree in $\tilde{x}_{1}$ than the second term, since $\tilde{x}_{1}$ is an endogenously bilinear mode. The inequality can therefore not hold as $\tilde{x}_{1} \rightarrow \infty$, since $\tilde{P}$ is positive definite. This point applies to all endogenously bilinear modes, not just open loop unstable ones.

For point 2), evaluate the controller for $\tilde{x}=\left[\begin{array}{ll}\tilde{x}_{1} & v^{T}\end{array}\right]^{T}$ for any finite, constant vector $v$, and let $\tilde{x}_{1} \rightarrow \infty$. Suppose the maximal degree of $\tilde{x}_{1}$ in $\tilde{c}_{0}(\tilde{x})$ is higher than the maximal degree of $\tilde{x}_{1}$ in $\tilde{c}_{1}(\tilde{x})$, then $u \rightarrow 0$ as $\tilde{x}_{1} \rightarrow \infty$. Then the stability is assessed with respect to the open loop dynamics (which correspond to an unstable mode) and leads to a contradiction. This argument applies to all open loop unstable modes, not just endogenously bilinear ones. 


\section{Remark 1}

Proposition 1 holds also for for systems with a diagonalizable $A$-matrix with complex-valued eigenvalues, since the eigenvalues and eigenvectors appear in complex conjugate pairs. Provided one uses the complex conjugate transpose of the vector $x$ when evaluating the Lyapunov function $V\left(\tilde{x}_{k+1}\right)=\tilde{x}_{k+1}^{T} \tilde{P} \tilde{x}_{k+1}$, the imaginary parts will cancel, and the proof above holds. The proof of Proposition 1 exploits the endogenously bilinear modes and thus the diagonalization of the bilinear part is instrumental. Consequently, the case when the $A$-matrix is not diagonalizable (contains a Jordan block) is not a trivial extension of the result.

\section{Remark 2}

Proposition 1 can be applied also to multiple input systems, if one assumes that the highest degree of $\tilde{x}_{1}$ in the first row of

$$
\tilde{B}_{\tilde{x}}\left[\tilde{c}_{1}(\tilde{x}) \quad \cdots \quad \tilde{c}_{m}(\tilde{x})\right]^{T}
$$

is always one degree higher than the maximal degree of $\tilde{x}_{1}$ in any $\tilde{c}_{i}(\tilde{x})$ (i.e., if one disregards the possibility that the maximal order terms may cancel when forming the product between $\tilde{B}_{\tilde{x}}$ and the controller numerator polynomials).

\section{CONTROLLER DESIGN METHOD}

Proposition 1 documents the need for a controller design procedure which is able to design rational polynomial state feedback controllers. This section addresses the systematic design of controllers of the form (4) to achieve stabilization of the system (2) to the origin by designing a controller which satisfies input constraints. However, the controller design described in this section does not require the $A$-matrix in (2) to be diagonalizable. The controller design is subject to control constraints of the form $\left|u_{i}(x)\right| \leq u_{i, \max }$.

\subsection{A scalarized Schur complement}

The Schur complement is often used in system analysis or controller design based on LMIs or SOS, as it can convert a non-linear relationship into an equivalent higher-dimensional linear one. However, for matrices there may be a significant difference between specifying $x^{T} Q(x) x>0$ and specifying that $Q(x)$ should be an SOS matrix - as the latter corresponds to demanding $z^{T} Q(x) z>0$ (where there is no relationship between $x$ and $z$ ).

It is therefore desirable to be able to retain scalar expressions when using the Schur complement. This can in some cases be done, as is shown by the following Lemma.

\section{Lemma 1}

Given a matrix

$$
M(x)=\left[\begin{array}{cc}
E(x) & H^{T}(x) \\
H(x) & P(x)
\end{array}\right] \in \mathbb{R}^{(n+r) \times(n+r)}
$$


with $P(x) \in \mathbb{R}^{r \times r}$ symmetric and invertible and $x \in \mathbb{R}^{n}$. Then

$$
\begin{gathered}
{\left[\begin{array}{ll}
x^{T} & z^{T}
\end{array}\right] M(x)\left[\begin{array}{l}
x \\
z
\end{array}\right]>0, \quad \forall(x, z) \neq(0,0)} \\
\text { is equivalent to } \\
x^{T}\left(E(x)-H^{T}(x) P^{-1}(x) H(x)\right) x>0, \forall x \neq 0 \text { and } z^{T} P(x) z>0, \forall z \neq 0
\end{gathered}
$$

Proof: This follows from the identity

$$
M(x)=\left[\begin{array}{cc}
I_{E} & H^{T}(x) P^{-1}(x) \\
0 & I_{P}
\end{array}\right]\left[\begin{array}{cc}
E(x)-H^{T}(x) P^{-1}(x) H(x) & 0 \\
0 & P(x)
\end{array}\right]\left[\begin{array}{cc}
I_{E} & 0 \\
P^{-1}(x) H(x) & I_{P}
\end{array}\right]
$$

where the subscripts on the identity matrices refer to the dimension of the matrices $E(x)$ and $P(x)$. Denote

$$
\left[\begin{array}{l}
x \\
w
\end{array}\right]=\left[\begin{array}{cc}
I_{E} & 0 \\
P^{-1}(x) H(x) & I_{P}
\end{array}\right]\left[\begin{array}{l}
x \\
z
\end{array}\right]
$$

and obtain the identity

$$
\left[\begin{array}{ll}
x^{T} & z^{T}
\end{array}\right] M(x)\left[\begin{array}{l}
x \\
z
\end{array}\right]=\left[\begin{array}{ll}
x^{T} & w^{T}
\end{array}\right]\left[\begin{array}{cc}
E(x)-H^{T}(x) P^{-1}(x) H(x) & 0 \\
0 & P(x)
\end{array}\right]\left[\begin{array}{l}
x \\
w
\end{array}\right]
$$

Whatever the value of $x$, a solution for $z$ of (10) can be found for any value of $w$, and vice versa.

\section{Remark 3}

Most of the proof above is very similar to the proof of the standard Schur complement. However, the key here is that one can pre- and postmultiply the matrix $M$ above with the appropriate vector, to obtain a scalar expression. This is not done in the standard Schur complement. While this extension to the standard Schur complement is mathematically very simple, its relevance in controller design will be illustrated in Section 5.

\subsection{SOS formulation}

This section addresses controller design, using controllers on the form (4), to optimize the region of quadratic stability. The denominator polynomial $c_{0}(x)$ will be assumed to be an SOS polynomial. However, there exists a possibility of using excessively large inputs, if all square terms in $c_{0}$ have roots accumulated in a small region of the state space. To guard against this situation, the denominator polynomial is specified as $c_{0}(x)=c_{0}^{\prime}(x)+1$, with $c_{0}^{\prime}(x)$ an SOS polynomial, thus ensuring that the denominator polynomial cannot approach zero anywhere in $\mathbb{R}^{n}$. Furthermore, in order to be able to apply the scalarized Schur complement, the controller is reformulated as

$$
u(x(k))=\frac{C(x(k)) x(k)}{c_{0}^{\prime}(x(k))+1} .
$$


with $C(x(k))$ a polynomial matrix. Note that $C(x(k))$ is not uniquely determined ${ }^{\dagger}$ by the polynomials $c_{i}(x(k))$, and a particular parametrization therefore will have to be chosen, but the product $C(x(k)) x(k)$ is indeed uniquely determined by the polynomials $c_{i}(x(k))$.

\section{Theorem 1}

Given a quadratic function $V(x)=x^{T} P x$, a scalar $\gamma>0$, polynomials $c_{i}(x), i \in[1, \ldots, m]$, and SOS polynomials $c_{0}^{\prime}(x)$ and $s_{1}(x, z)$, a bilinear discrete time system (3) in closed loop with the control law (4) is stable $\forall x$ such that $x^{T} P x<\gamma$, provided

$$
\left[\begin{array}{ll}
x^{T} & z^{T}
\end{array}\right] M(x)\left[\begin{array}{l}
x \\
z
\end{array}\right]-s_{1}(x, z)\left(\gamma-x^{T} P x\right)>0
$$

where

$$
M(x)=\left[\begin{array}{cc}
\left(c_{0}^{\prime}(x)+1\right) P & \left(\left(c_{0}^{\prime}(x)+1\right) A+\left(B_{x}+B\right) C(x)\right)^{T} P \\
P\left(\left(c_{0}^{\prime}(x)+1\right) A+\left(B_{x}+B\right) C(x)\right) & \left(c_{0}^{\prime}(x)+1\right) P
\end{array}\right]
$$

Proof: Dividing (12) with the strictly positive $\left(c_{0}^{\prime}(x)+1\right)$, and noting that $\frac{s_{1}(x, z)}{c_{0}^{\prime}(x)+1}\left(\gamma-x^{T} P x\right)$ is positive $\forall x \neq 0$ with $x^{T} P x<\gamma$, one may conclude that

$$
\left[\begin{array}{ll}
x^{T} & z^{T}
\end{array}\right] \frac{1}{\left(c_{0}^{\prime}(x)+1\right)} M(x)\left[\begin{array}{l}
x \\
z
\end{array}\right]>0
$$

for all $x \neq 0$ with $x^{T} P x<\gamma$. Considering the controller in (11), the bilinear system dynamics in (3) and Lemma 1, it can then be concluded that

$$
x(k)^{T} P x(k)-x(k+1)^{T} P x(k+1)-\frac{s_{1}(x(k), z)}{c_{0}^{\prime}(x(k))+1}\left(\gamma-x(k)^{T} P x(k)\right)>0 \forall x(k) \neq 0
$$

(plus the trivial consequence that $z^{T} P z>0$ ), and hence the Lyapunov function decreases $\forall x(k) \neq 0$ with $x^{T}(k) P x(k)<\gamma$.

\section{Theorem 2}

Given the polynomial $c_{i}(x)$, SOS polynomials $c_{0}^{\prime}(x)$ and $q_{i}(x)$, the input constraint is satisfied $\forall x$ with $x^{T} P x<\gamma$ provided

$$
\left[\begin{array}{cc}
\left(c_{0}^{\prime}(x)+1\right) u_{\max , i}^{2}-q_{i}(x)\left(\gamma-x^{T} P x\right) & c_{i}(x) \\
c_{i}(x) & c_{0}^{\prime}(x)+1
\end{array}\right]>0
$$

Proof: Following the same approach as in the proof of Theorem 1, it can be shown that (15) is equivalent to

$$
u_{m a x, i}^{2}-u_{i}^{2}(x)-\frac{q_{i}(x)}{\left(c_{0}^{\prime}(x)+1\right)}\left(\gamma-x^{T} P x\right)>0,
$$

and hence $u_{\text {max }, i}^{2}-u_{i}^{2}(x)>0 \forall x \in\left\{x \mid x^{T} P x<\gamma\right\}$.

If the polynomial $c_{i}(x(k))$ contains a term $\hat{c}_{m n} x_{m}(k) x_{n}(k)$, row $i$ of $C(x(k))$ may contain the element $\hat{c}_{m n} x_{m}$ in column $n$, or the element $\hat{c}_{m n} x_{n}(k)$ in column $m$. 


\subsection{Optimization formulation}

Theorems 1 and 2 allow for controller design according to

$$
\max _{\dot{c}_{0}(x), c_{i}(x), s_{1}(x, z), q_{i}(x), P \gamma}
$$

subject to : constraints (12) and (15), $\dot{c}_{0}(x), s_{1}(x, z), q_{i}(x)$ SOS,

$$
P>0, \operatorname{trace}(P)=\text { constant }
$$

The final constraint in (17) is a normalizing constraint included in order to avoid both $\gamma$ and $P$ growing without bound - without describing a larger quadratic stability region.

There are several bilinear terms in (17). With access to an optimization solver handling bilinear constraints, (17) may be solved directly. Here it is instead proposed to iteratively fix some variables and solve for the other variables, which appears to be a common approach to solving bilinear SOS (see, e.g., [6]). Algorithm 1 describes the resulting controller design procedure.

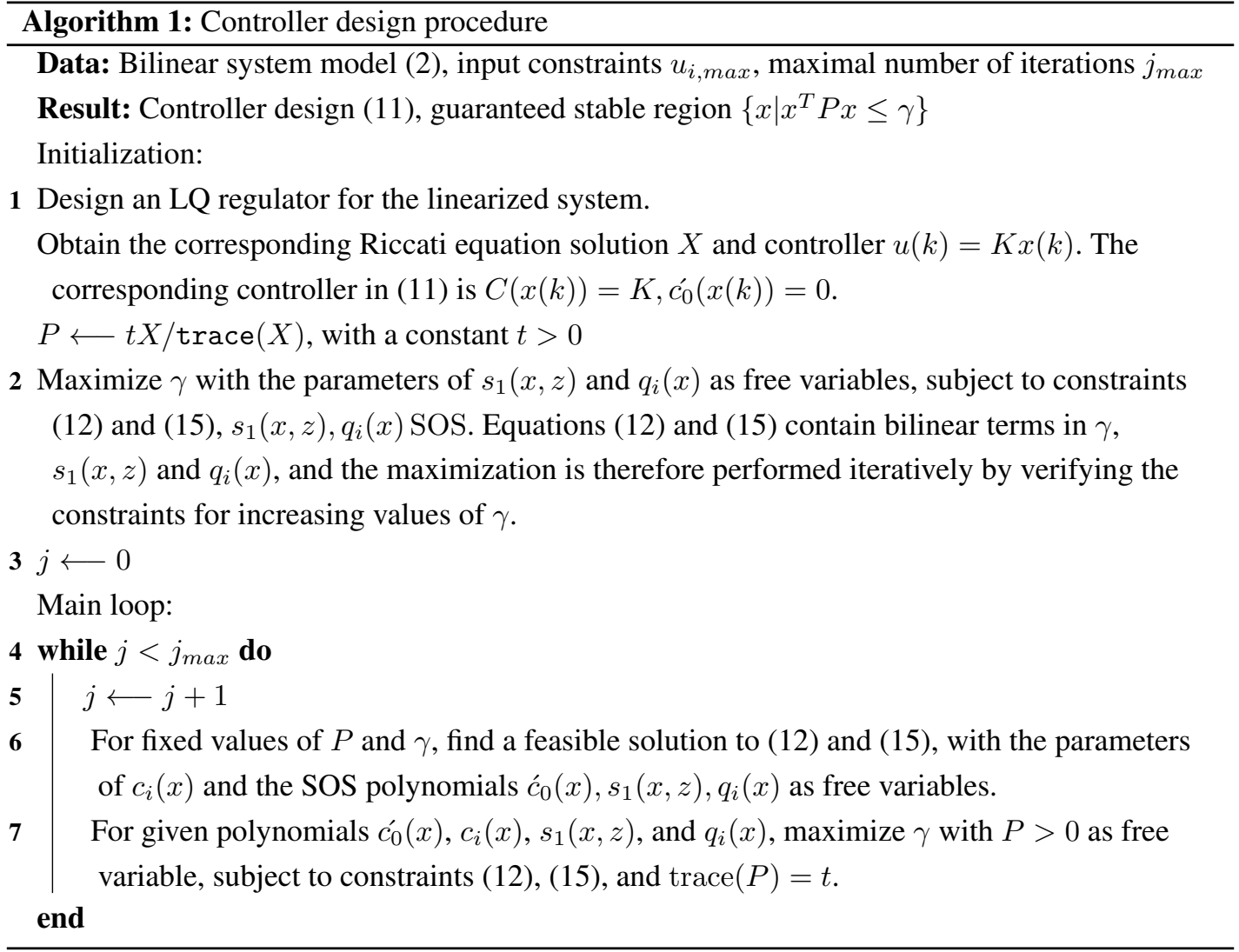

Note that semidefinite solvers typically return solutions in the analytic center of the feasible region [31]. Finding a feasible solution in step 6 above therefore provides room for further optimization in step 7.

Although numerical experience with this approach is good, there is no formal proof that this iteration will (in the limit) lead to the maximum region of convergence for a rational polynomial controller with a quadratic Lyapunov function. Note, however, that step 7 above can easily be modified such that the new region of convergence always contains the region of convergence from the previous iteration. 


\subsection{Improving rate of convergence}

It is well known that maximizing the region of convergence leads to rather slow control, in particular near the boundary of the region in question. To improve the rate of convergence, a certain decrease in Lyapunov function in each step can be imposed by requiring that

$$
x(k)^{T} P x(k)-x(k+1)^{T} P x(k+1)>\alpha x(k)^{T} P x(k)
$$

for some $\alpha, 0<\alpha<1$. This changes element $(1,1)$ of matrix $M(x)$ in (12) and (13) to $M_{11}(x)=$ $(1-\alpha)\left(c_{0}^{\prime}(x)+1\right) P$.

\section{Remark 4}

The controller design approach in this section does not explicitly take into account Proposition 1, although it can be used to guide the selection of the degrees of the controller polynomials. However, Proposition 1 is concerned with global stabilization, thus if stabilization in a bounded region of the state space is the aim, the polynomial degrees may still be a degree of freedom in the design.

\section{NUMERICAL EXAMPLES}

This section will apply the controller design method described above to three examples. In all three examples, the system studied is open loop unstable, making the controllers proposed in [15], [16] and [17] inapplicable.

Example 1: In the following, a second-order bilinear system, proposed initially in [24], is considered:

$$
A=\left[\begin{array}{cc}
1 & 0.01 \\
0.01 & 1
\end{array}\right], B_{1}=\left[\begin{array}{cc}
0.001 & 0 \\
0 & -0.004
\end{array}\right], b_{1}=\left[\begin{array}{l}
0.09 \\
0.09
\end{array}\right]
$$

The input is constrained to $|u| \leq 2$. The problem to be solved is the determination of the controller which stabilizes the system in the maximum possible region of $x_{k}^{T} P x_{k}<\gamma . P$ is considered as identity matrix.

First, the region of convergence is maximized while keeping $P$ fixed. The highest order considered in the controller polynomials is $n_{p}=2$. The maximum region where YALMIP could find a controller to stabilize the system is given by $\gamma=295$. This should be compared to the value $\gamma=150$ obained in [27]. The difference is due to the use of the scalarized Schur complement in the present work. The designed controller based on (4) is as follows:

$$
\begin{aligned}
& c_{1}\left(x_{k}\right)=-0.0838 x_{1}-0.1586 x_{2}-0.0002 x_{1}^{2}+0.0046 x_{1} x_{2}-0.0061 x_{2}^{2} \\
& c_{0}\left(x_{k}\right)=1.0959-0.0018 x_{1}-0.0029 x_{2}+0.0044 x_{1}^{2}-0.0046 x_{1} x_{2}+0.0053 x_{2}^{2}
\end{aligned}
$$

The state evolution in time, input and cost function for designed controller are shown in Fig. 1 for the initial state of $x_{0}=[-10,13.9]^{T}$. Note that, although (13) cannot be verified for $\gamma>295$, this does not mean that the system is necessarily unstable in that region. 


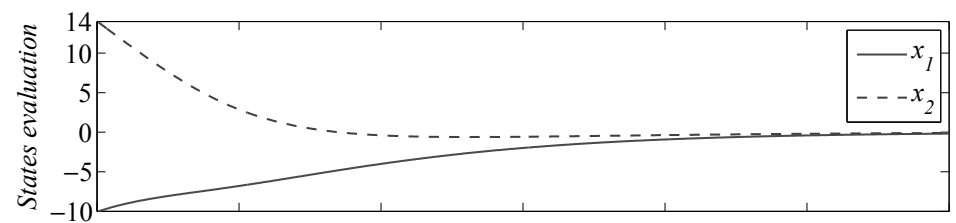

(a)

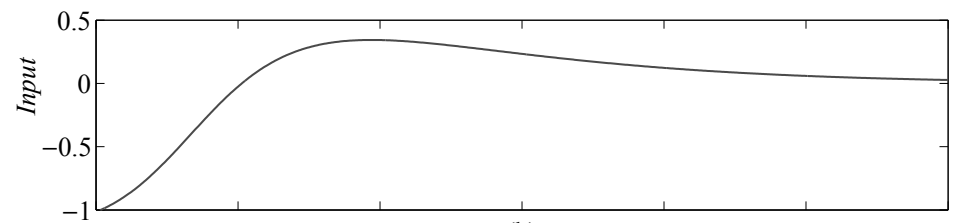

(b)

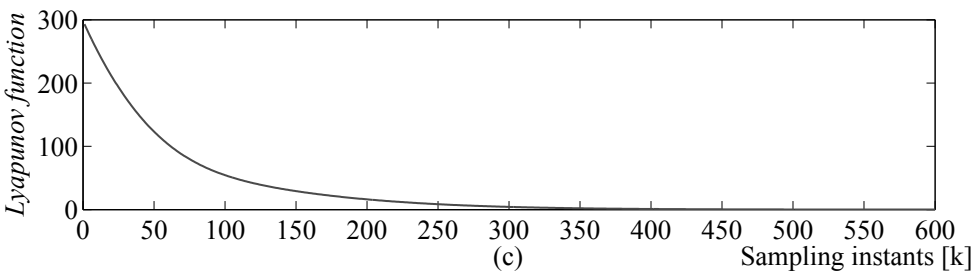

Figure 1. Simulation results for example 1 system controlled by SOS method: (a) states, (b) input, and (c) cost function

In Fig. 2, the phase portrait of the closed loop system for initial states belonging to the $x_{1}^{2}+x_{2}^{2}=$ 295 is depicted.

\section{Remark 5}

The problem formulation in [24] includes the state constraints $\left|x_{i}\right| \leq 4, i \in\{1,2\}$, which makes the objective of the controller design different from the one in the present paper. Nevertheless, Fig. 2 shows that the controller presented here practically makes the set $\left\{x|| x_{1}|\leq 4,| x_{2} \mid \leq 4\right\}$ positively invariant, and thus that the state constraints are fulfilled for any initial condition within this set.

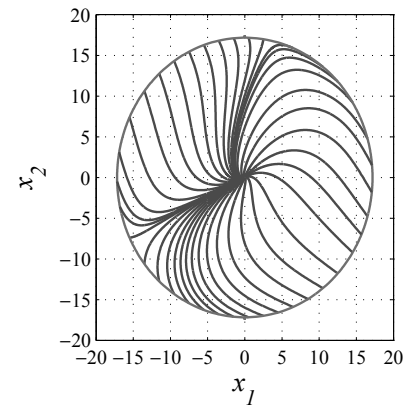

(a)

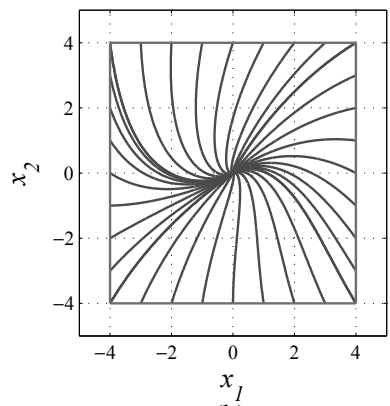

(b)

Figure 2. State trajectories for example 1: (a) starting from the border of the maximum region of stability (b) starting from $\left|x_{i}\right| \leq 4$ as in [24]

To improve the rate of convergence, the controller design is performed while specifying $\alpha=$ 0.015 in (18). Note that by adding $\alpha$ to the problem, the maximum region of convergence will 
decrease. In this example, it decreases to $\gamma=122$. The designed controller is as follows:

$$
\begin{aligned}
& c_{1}\left(x_{k}\right)=-0.1022 x_{1}-0.1268 x_{2}+0.0008 x_{1}^{2}+0.0015 x_{1} x_{2}-0.0052 x_{2}^{2} \\
& c_{0}\left(x_{k}\right)=1.0039+0.0002 x_{1}-0.0007 x_{2}+0.0008 x_{1}^{2}+0.0001 x_{1} x_{2}+0.0008 x_{2}^{2}
\end{aligned}
$$

The responses of the system for both controllers (for $\alpha=0$ and $\alpha=0.015$ ) are shown in Fig. 3, which shows that by adding the term $\alpha$, the rate of convergence is increased.

Finally, the guaranteed stable region is increased using the iterative procedure described in Section 4.3, starting with $P=I$. Figure 4 shows the initial region of convergence, and the region of convergence obtained after 15 iterations.

Example 2: Consider the third-order bilinear system with two inputs found in [24]:

$$
\begin{gathered}
A=\left[\begin{array}{ccc}
1.10 & -0.2 & -0.34 \\
-0.06 & 0.7 & -0.42 \\
0.41 & 0.41 & 0.90
\end{array}\right], b_{1}=\left[\begin{array}{c}
3.75 \\
1.05 \\
-0.85
\end{array}\right], b_{2}=\left[\begin{array}{c}
0 \\
-1.33 \\
-0.49
\end{array}\right] \\
B_{1}=\left[\begin{array}{ccc}
-0.12 & -0.22 & 0.36 \\
-0.32 & 0.48 & 0.36 \\
-0.35 & 0.36 & -0.18
\end{array}\right], B_{2}=\left[\begin{array}{ccc}
-0.18 & 0.30 & 0.07 \\
-0.03 & -0.18 & -0.38 \\
0.55 & -0.74 & -0.77
\end{array}\right]
\end{gathered}
$$

Both control inputs have to respect the linear constraints $-1 \leq u_{i} \leq 1$. The matrix $P$ in the cost

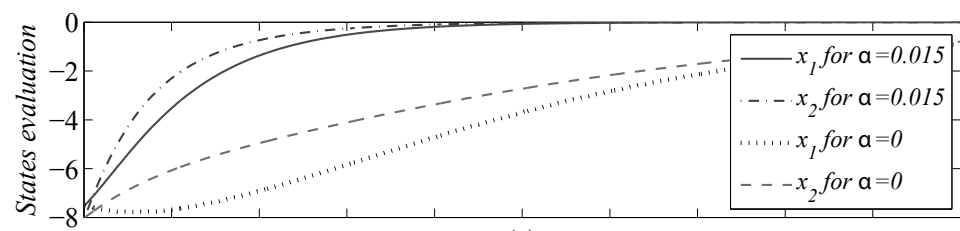

(a)

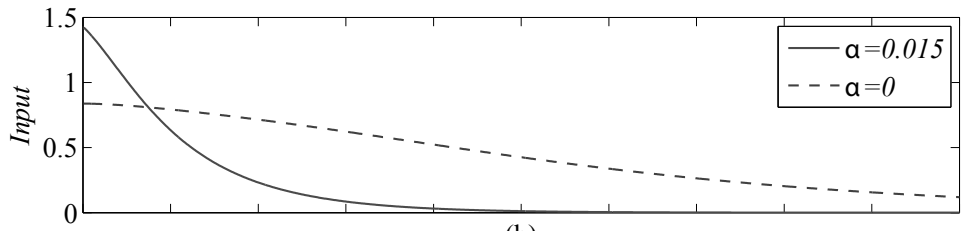

(b)

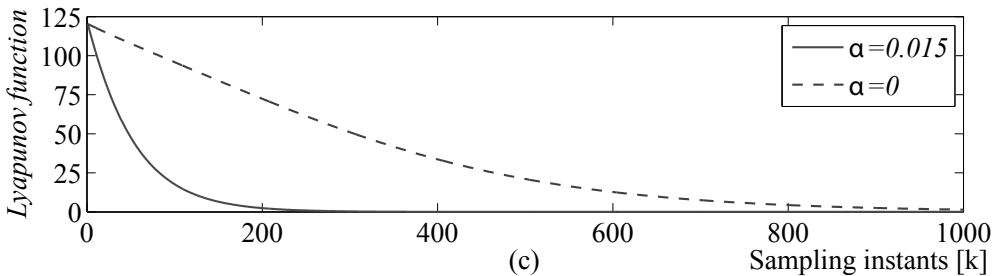

Figure 3. Improvement of the response in example 1 by specifying $\alpha=0.015,(\gamma=120)$ : (a) states, (b) input, and (c) cost function

function is chosen as:

$$
P=\left[\begin{array}{ccc}
2 & 0.1 & 0.1 \\
0.1 & 1.5 & 0.1 \\
0.1 & 0.1 & 1
\end{array}\right]
$$




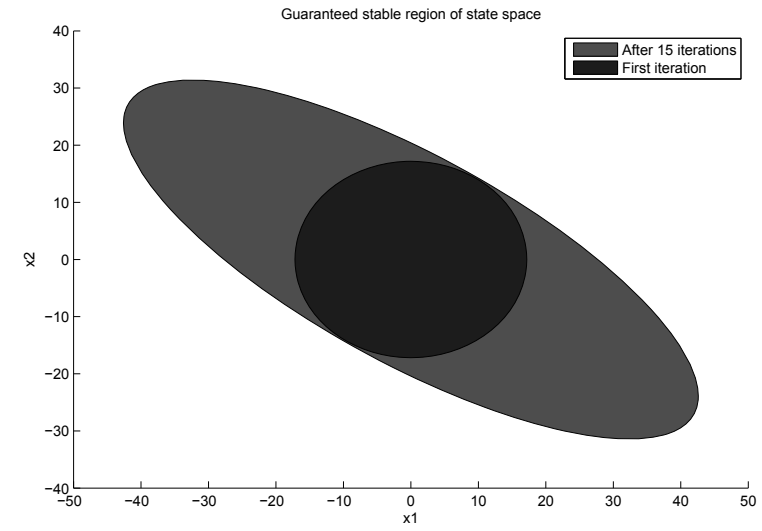

Figure 4. Initial region of convergence and region of convergence after 15 iterations

Using SOS programming, keeping $P$ fixed, a region of stability parametrized by $\gamma=33$ results. The value obtained in [27] was $\gamma=4$, again showing the advantage of using the scalarized Schur complement. The designed controller is:

$$
\begin{aligned}
c_{1}\left(x_{k}\right)= & -0.1064 x_{1}-0.0002 x_{2}+0.0657 x_{3}-0.0043 x_{1}^{2} \\
& -0.0052 x_{1} x_{2}+0.0026 x_{2}^{2}+0.0105 x_{1} x_{3}+0.0028 x_{2} x_{3}-0.0067 x_{3}^{2} \\
c_{2}\left(x_{k}\right)= & -0.0012 x_{1}+0.0105 x_{2}-0.0441 x_{3}+0.0042 x_{1}^{2} \\
& -0.0012 x_{1} x_{2}+0.0049 x_{2}^{2}-0.0114 x_{1} x_{3}-0.0011 x_{2} x_{3}+0.0113 x_{3}^{2} \\
c_{0}\left(x_{k}\right)= & 1.0061-0.0012 x_{1}-0.0014 x_{2}+0.0044 x_{3} \\
& +0.0158 x_{1}^{2}-0.0012 x_{1} x_{2}+0.0219 x_{2}^{2}+0.0068 x_{1} x_{3}+0.0057 x_{2} x_{3}+0.0045 x_{3}^{2}
\end{aligned}
$$

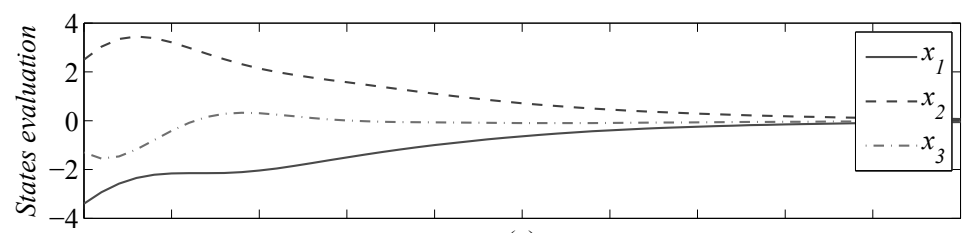

(a)

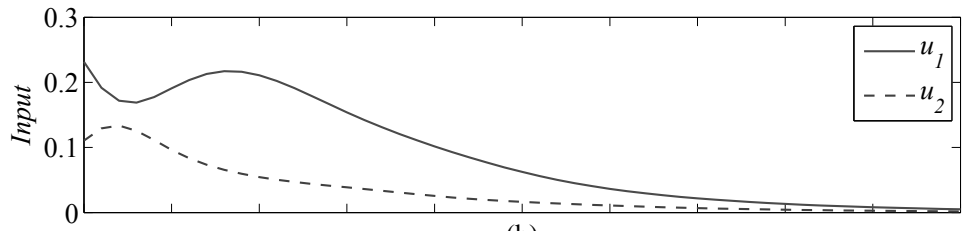

(b)

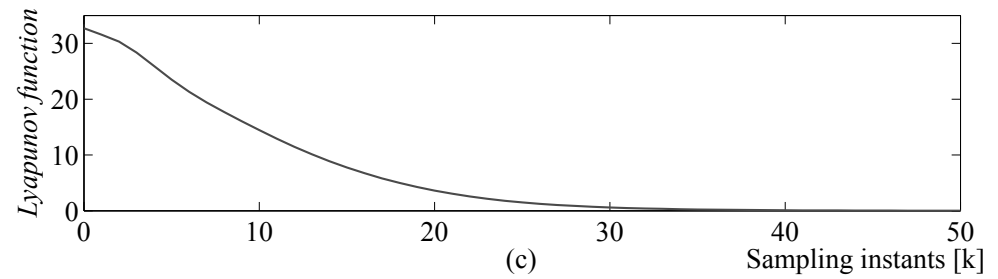

Figure 5. Simulation results for example 2 system controlled by SOS method: (a) states, (b) input, and (c) cost function 


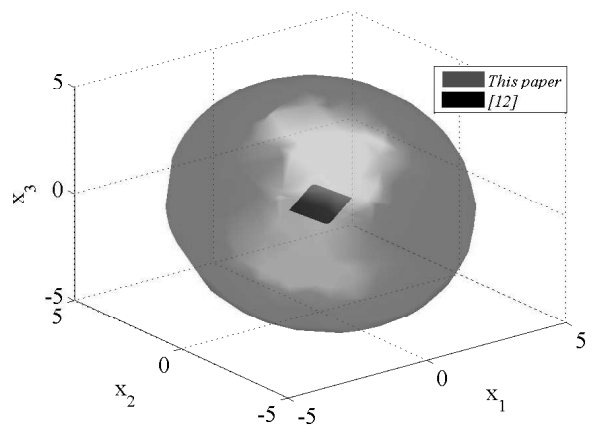

Figure 6. Region of stability calculated for example 2. Light grey: SOS design, dark grey: the design in [24]

The state responses for the calculated controller for the initial state $x_{0}=[-3.4,2.5,-1.3]^{T}$ is depicted in Fig. 5 along with input and cost function.

The region of quadratic stability $\left(x_{k}^{T} P x_{k}<\gamma\right)$ calculated for this example is shown in Fig. 6 in light (transparent) grey. In [24] an optimization problem is solved to maximize the region of convergence, using a problem formulation involving polyhedral Lyapunov functions. The resulting region of convergence is shown in Fig. 6 in dark grey for comparison.

Example 3: Consider the following second order bilinear system [23]:

$$
A=\left[\begin{array}{ll}
0.8 & 0.5 \\
0.4 & 1.2
\end{array}\right], B_{1}=\left[\begin{array}{cc}
0.45 & 0.45 \\
0.3 & -0.3
\end{array}\right], b_{1}=\left[\begin{array}{l}
1 \\
2
\end{array}\right]
$$

The input is constrained to $|u| \leq 0.5$. The problem to be solved is the determination of the controller which stabilizes the system in the maximum possible region of $x_{k}^{T} P x_{k}<\gamma$. The matrix $P$ is chosen as

$$
P=\left[\begin{array}{ll}
1 & 1 \\
1 & 2
\end{array}\right]
$$

Solving the problem in YALMIP for maximum $\gamma$ results in $\gamma=6$. The designed controller is as follows:

$$
\begin{aligned}
& c_{1}\left(x_{k}\right)=-0.1733 x_{1}-0.2312 x_{2}+0.0129 x_{1}^{2}+0.0176 x_{1} x_{2}-0.0024 x_{2}^{2} \\
& c_{0}\left(x_{k}\right)=1.0051+0.0073 x_{1}+0.0002 x_{2}+0.0070 x_{1}^{2}-0.0005 x_{1} x_{2}+0.0062 x_{2}^{2}
\end{aligned}
$$

State responses, input and cost function evolution in time is depicted in Fig. 7. In addition, the calculated region of convergence for SOS method is shown in Fig. 8. This problem is also solved in [23] using polyhedral Lyapunov functions and calculated region of convergence is also shown in the same figure for comparison. In this example, the value $\gamma=6$ obtained is the same as in what was obtained in [27]. However, increasing the allowable input to $|u| \leq 2.0$ increases $\gamma$ to 7.5 for the approach in [27], whereas for the approach in this paper one obtains $\gamma=11.1$. Note that the scalarized Schur complement is not used in Thm. 2 which addresses input constraints. Relaxing the input constraint therefore increases the importance of utilizing the scalarized Schur complement in Thm. 1. 


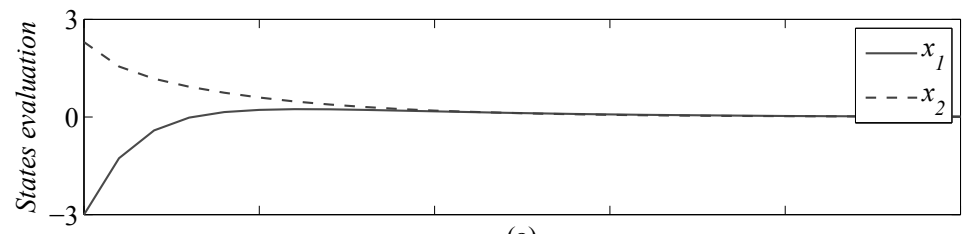

(a)

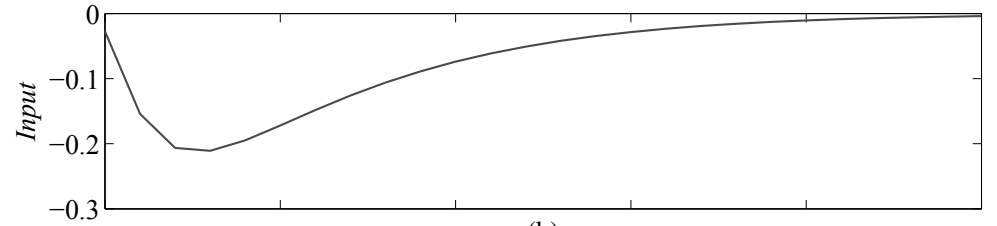

(b)

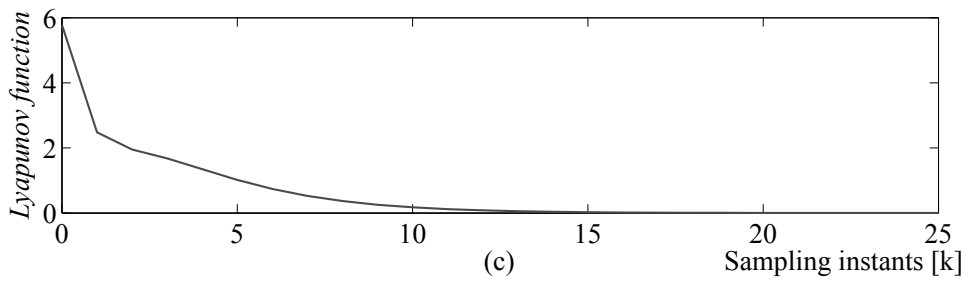

Figure 7. Simulation results for example 3 system controlled by SOS method: (a) states, (b) input, and (c) cost function

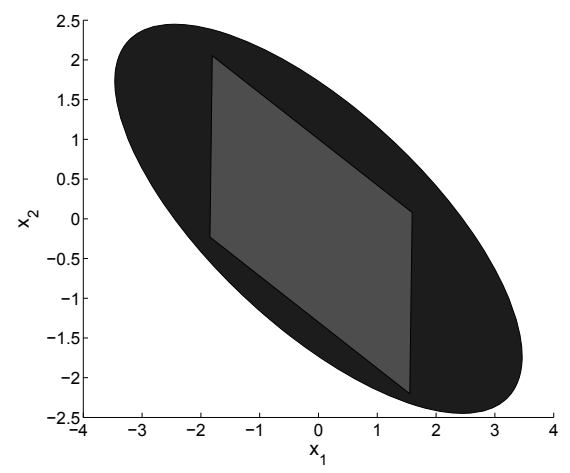

Figure 8. Region of convergence calculated for example 3 using polyhedral Lyapunov function in [23] in light grey and using SOS method in dark grey

\section{ACKNOWLEDGMENT}

Helpful discussions with Dr. Nikos Athanasopoulos from the Eindhoven University of Technology are gratefully acknowledged.

\section{CONCLUSIONS}

Conditions for global quadratic stability of discrete-time bilinear systems controlled by rational polynomial controllers are studied. It is shown that the denominator polynomial and numerator polynomials should have the same maximal degree in any state representing an unstable endogenously bilinear mode. 
A scalarized version of the Schur complement is presented, and this is used in formulating optimization based conditions for controller design. Comparing results of the examples in this paper with those in [27], it is found that using the scalarized Schur complement resulted in significant enlargement of the stable region in two out of three cases. In the third case, a severe input constraint was more important than the conservatism of not using the scalarized Schur complement - and relaxing the input constraint again allowed the scalarized Schur complement to provide an enlargement of the stable region.

Optimization formulations for controller design based on SOS programming are given, both for maximizing the region of convergence and for imposing a specified rate of convergence within a given region of convergence.

The controller design is not applicable to systems such as Example 2 in [32] with the parameter $\lambda=0$. In that example, the origin is on the border of the stabilizable region, and no continuous Lyapunov function can be used to prove stability. Note also that the stability of the origin in such a system is not robust, even infinitesimal disturbances may be sufficient to drive the system into the un-stabilizable region.

SOS-based controller design are known to rapidly become computationally demanding with increasing system size. The largest system for which the proposed design method has been successfully handled by the authors has 7 states and 5 inputs. This should be larger than many systems of engineering interest, for further details see [33]. Current research exploiting sparsity patterns in SOS calculations bear the promise of enabling larger systems to be handled [34].

\section{REFERENCES}

1. Parrilo PA. Structured Semidefinite Programs and Semialgebraic Geometry Methods in Robustness and Optimization. PhD Thesis, California Institute of Technology 2000.

2. Hancock EJ, Papachristodoulou A. Generalised absolute stability and sum of squares. Automatica 2013; 49(4):960967.

3. Chesi G. Domain of Attraction. Analysis and control via SOS programming, Lecture Notes in Control and Information Sciences, vol. 415. Springer Verlag: London, GB, 2011.

4. Valmòrbida G, Tarbouriech S, Garcia G. Design of polynomial control laws for polynomial systems subject to actuator saturation. IEEE Transactions on Automatic Control 2013; :1758-1770.

5. Zheng Q, Wu F. Regional stabilization of polynomial non-linear systems using rational Lyapunov functions. International Journal of Control; 82(9):1605-1615.

6. Valmòrbida G, Tarbouriech S, Garcia G, Zaccarian L. Synthesis of polynomial static state feedback laws for discrete-time polynomial systems with saturating inputs. Proceedings of the American Control Conference, 2012; $2325-2329$.

7. Oliveira MZ, Gomes da Silva Jr JM, Coutinho D. Regional stabilization of rational discrete-time systems with magnitude control constraints. Proceedings of the American Control Conference, 2013; 241-246.

8. Spinu V, Athanasopoulos N, Lazar M, Bitsoris G. Stabilization of Bilinear Power Converters by Affine State Feedback Under Input and State Constraints. IEEE Transactions on Circuits and Systems II: Express Briefs Aug 2012; 59(8):520-524, doi:10.1109/TCSII.2012.2204842.

9. Lamoudi MY, Alamir M, Béguery P, et al.. Unified NMPC for multi-variable control in smart buildings. IFAC 18th World Congress, Milano, Itlay, 2011.

10. Gutman PO. Stabilizing controllers for bilinear systems. IEEE Transactions on Automatic Control Aug 1981; 26(4):917-922, doi:10.1109/TAC.1981.1102742.

11. Nobuyama E, Aoyagi T, Kami Y. A Sum of Squares Optimization Approach to Robust Control of Bilinear Systems, ser. Recent Advances in Robust Control - Theory and Applications in Robotics and Electromechanics. InTech, 2011; 39-54, doi:10.5772/709. 
12. Kang D, Won S, Jang YJ. Guaranteed cost control for bilinear systems by static output feedback. Applied Mathematics and Computation 2013; 219(14):7398-7405.

13. Elliott DL. Bilinear Control Systems. Matrices in Action, Applied Mathematical Sciences, vol. 169. Springer: Heidelberg, Germany, 2009.

14. Pardalos PM, Yatsenko V. Optimization and control of bilinear systems, theory, algorithms, and applications. Springer: New York, NY, USA, 2008.

15. Lin W, Byrnes C. KYP lemma, state feedback and dynamic output feedback in discrete-time bilinear systems. Systems \& Control Letters 1994; :127-136.

16. Chen MS, Hwang YR, Huang KC. Nonlinear controls for a class of discrete-time bilinear systems. International Journal of Robust and Nonlinear Control Sep 2003; 13(11):1079-1090, doi:10.1002/rnc.805.

17. Chiou J, Kung FC, Li THS. Robust stabilization of a class of singularly perturbed discrete bilinear systems. IEEE Transactions on Automatic Control 2000; :1187-1191.

18. Kim BS, Kim YJ, Lim MT, Kim B. Stabilizing control for discrete time multi-input bilinear systems. Proceedings of the 15th IFAC Wolrd Congress, 2002.

19. Lu G, Feng G, Jiang ZP. Saturated feedback stabilization of discrete-time descriptor bilinear systems. IEEE Transactions on Automatic Control 2007; 52(9):1700-1704.

20. Tang GY, Ma H, Zhang BL. Successive-approximation approach of optimal control for bilinear discrete-time systems. IEE Proc. Control Theory \& Applications 2005; :637-644.

21. Fontes AB, Dorea CE, da S Garcia MR. An iterative algorithm for constrained MPC with stability of bilinear systems. 2008 16th Mediterranean Conference on Control and Automation, IEEE, 2008; 1526-1531, doi: 10.1109/MED.2008.4602048.

22. Bloemen H, Cannon M, Kouvaritakis B. Interpolation in MPC for discrete time bilinear systems. Proceedings of the 2001 American Control Conference, vol. 5, 2001; 3061-3066, doi:10.1109/ACC.2001.946386.

23. Bitsoris G, Athanasopoulos N. Constrained Stabilization of Bilinear Discrete-Time Systems Using Polyhedral Lyapunov Functions. 17th World Congress of the International Federation of Automatic Control, 2008; 2502-2507.

24. Athanasopoulos N, Bitsoris G. Unconstrained and constrained stabilisation of bilinear discrete-time systems using polyhedral Lyapunov functions. International Journal of Control Dec 2010; 83(12):2483-2493, doi: 10.1080/00207179.2010.531396.

25. Athanasopoulos N, Bitsoris G, Vassilaki M. Ultimate boundedness and robust stabilization of bilinear discretetime systems. IEEE Conference on Decision and Control and European Control Conference, 2011; 4622-4627, doi:10.1109/CDC.2011.6161233.

26. Bacic M, Cannon M, Kouvaritakis B. Constrained control of SISO bilinear systems. IEEE Transactions on Automatic Control Aug 2003; 48(8):1443-1447.

27. Vatani M, Hovd M, Olaru S. Control design and analysis for discrete time bilinear systems using sum of squares methods. Proceedings of the 53rd IEEE Conference on Decision and Control, 2014; 3143-3148.

28. Löfberg J. Yalmip : A toolbox for modeling and optimization in MATLAB. Proceedings of the CACSD Conference, Taipei, Taiwan, 2004. URL http://control.ee.ethz.ch/ joloef/yalmip.php.

29. Löfberg J. Pre- and Post-Processing Sum-of-Squares Programs in Practice. IEEE Transactions on Automatic Control May 2009; 54(5):1007-1011, doi:10.1109/TAC.2009.2017144.

30. Jiang ZP, Wang Y. Input-to-state stability for discrete-time nonlinear systems. Automatica 2001; 37:857-869.

31. Löfberg J. Strictly feasible sum-of-squares. https://yalmip.github.io/Strictly-feasible-sum-of-squares/.

32. Tie L. On controllability of driftless inhomogeneous bilinear systems. 53rd IEEE Conference on Decision and Control, Los Angeles, California, USA, 2014; 3161-3166.

33. Vatani M, Hovd M, Saeedifard M. Control of the modular multilevel converter based on a discrete-time bilinear model using the sum of squares decomposition method. IEEE Transactions on Power Delivery 2015; 30(5):21792188.

34. Ahmadi AA, Parrilo PA. Towards scalable algorithms with formal guarantees for lyapunov analysis of control systems via algebraic optimization. Proceedings of the 53rd IEEE Conference on Decision and Control, 2014; 2272-2281. 